Running head: STRESS, STARTLE AND PPI

\title{
Effect of Stress and Attention on Startle Response and Prepulse Inhibition
}

\author{
Luis Gonzalo De la Casa, Auxiliadora Mena, Juan Carlos Ruiz-Salas
}

Dpt. Experimental Psychology

Seville University (Spain)

Corresponding author:

Luis G. De la Casa

Dpt. Psicología Experimental

Facultad de Psicología

C/ Camilo José Cela, s/n

41018 Sevilla (Spain)

Tel. (34) 954557682 Fax: (34) 954551784

E-mail: delacasa@us.es 


\begin{abstract}
The startle reflex magnitude can be modulated when a weak stimulus is presented before the onset of the startle stimulus, a phenomenon termed Prepulse Inhibition (PPI). Previous research has demonstrated that emotional processes can modulate PPI and startle intensity, but the available evidence is inconclusive. In order to obtain additional evidence in this domain, we conducted two experiments intended to analyze the effect of induced stress and attentional load on PPI and startle magnitude. Specifically, in Experiment 1 we used a between subject strategy to evaluate the effect on startle response and PPI magnitude of performing a difficult task intended to induce stress in the participants, as compared to a group exposed to a control task. In Experiment 2 we evaluated the effect of diverting attention from the acoustic stimulus on startle and PPI intensity. The results seem to indicate that induced stress can reduce PPI, and that startle reflex intensity is reduced when attention is directed away from the auditory stimulus that induces the reflex.
\end{abstract}

Key Words: Startle; Prepulse Inhibition; Stress; Attention 


\section{Introduction.}

The startle reflex is an involuntary response consisting of flexion of certain muscle groups, most marked in the upper half of the body, that is produced when an intense stimulus appears. From a functional point of view, this reflex serves as a protective function against any signal strong enough to indicate a circumstance which might endanger the life or integrity of the individual, since it provides fast muscle activation that can support a defensive response if necessary (Blumenthal, 2015; Dawson, Schell, \& Böhmelt, 1999).

Even though the startle response represents a seemingly unalterable reflex reaction, there are several circumstances that may modulate its intensity, either by intensifying or reducing the reflex response. Thus, for example, the startle reflex increases when a sensitization process (e.g., Peeke, \& Petrinovich, 1984) or a prepulse facilitation process (e.g., Wynn, Dawson, Schell, McGee, Salveson, \& Green, 2004 ) is induced. Conversely, a reduced response is observed after stimulus habituation (e.g., Pilz \& Schnitzler, 1996) or prepulse inhibition (e.g., Hoffman \& Searle, 1968; Graham, 1975).

The startle modulation process that has probably received the most attention in the recent scientific literature is the so-called Pre-Pulse Inhibition (PPI), a phenomenon that was operationally defined in 1965 by Hoffman and Searle as the reduced startle reflex to an intense sound (named Pulse) that appears when it is preceded by a weaker sound (named Prepulse) presented between 30 to 500 ms before the Pulse. Since the phenomenon was described for the first time, a large amount of research has been conducted intended to analyze PPI from physiological, psychological, or even psychiatric perspectives (see, for reviews, Blumenthal, 2015; Braff, Geyer, \& Swerdlow, 2001; Larrauri \& Schmajuk, 2006).

From a functional perspective, Graham (1975) proposed that PPI has the purpose of protecting the processing of current information. Specifically, a preattentional brain inhibitory process intended to prevent current processing from 
interference will be active until the attended stimulus is fully processed. Such inhibition impedes the interference that would be induced by mobilization of the attentional resources that usually occurs when new stimuli are detected, and the intensity or the nature of the stimulus that follows is independent of the stimulus that is currently being processed. This PPI interpretation has been complemented by a physiological perspective proposing that any new stimulus presentation activates an inhibitory process involving limbic cortico-striato-pallido-pontine circuitry that minimizes the processing of other stimuli during a "gate" that ranges from 30 to $500 \mathrm{~ms}$ (e.g., Schmajuk, Larrauri, De la Casa, \& Levin, 2009). From this perspective, PPI is considered to reflect the functioning of a central process, that has been labeled sensorimotor gating, that is responsible for protecting the processing of the first stimulus (the Prepulse) from the interference of other incoming stimuli (e.g., Swerdlow, Braff, \& Geyer, 1999; Swerdlow, Caine, Braff, \& Geyer, 1992). Since the integrity of the sensorimotor gating process ensures an adequate organization of our cognitive resources, it has been proposed that PPI can be employed as a neurobiological marker for those pathologies characterized by inadequate motor or sensory gating such as, for example, schizophrenia (e.g., Braff, Grillon, \& Geyer, 1992; Gottesman \& Gould, 2003; Light, Swerdlow, Rissling, Radant, Sugar, Sprock, Pela, Geyer, \& Braff, 2012; Turetsky, Calkins, Light, Olincy, Radant, \& Swerdlow, 2007). Also, PPI deficits have been reported in cases of obsessive-compulsive disorder (e.g. Hoenig, Hochrein, Quednow, Maier, \& Wagner, 2005), patients with Huntington's disease (Swerdlow, Paulsen, Braff, Butters, Geyer, \& Swenson, 1995), and many other pathologies (see, for a recent review, Kohl, Heekeren, Klosterkötter, \& Kuhn, 2013). In fact, the relationship between PPI and psychopathology has largely favored the use of such phenomena as an experimental paradigm in psychophysiological research and, particularly, in the field of study of psychiatric disorders (Dahmen \& Corr, 2004). Since a common factor in many psychopathologies is the existence of high anxiety levels, this should be a relevant aspect to be specially considered when 
analyzing startle reflexes or PPI in pathological populations (e.g., Grillon \& Baas, 2003; Huppert, Weiss, Lim, Pratt, \& Smith, 2001). In fact, there is experimental evidence indicating that the induction of positive or negative emotional states in participants without pathologies during startle or PPI induction modulates both responses (e.g., De la Casa, Mena, \& Puentes, 2014; Vrana, Spence, \& Lang, 1988).

More specifically, evidence on startle response and PPI changes induced by high anxiety or stress states have been obtained in experiments both with animals and with human participants, but the results seem contradictory. Thus, for instance, Leitner (1986) found disrupted PPI in rats submitted to stress induced by a forced swim procedure, and Pijlman, Herremans, van de Kieft, and van Ree (2003) found the same results in rats receiving a foot-shock treatment, but intact PPI was found in rats submitted to psychological stress (by being witnesses to the shock treatment). In experiments with human participants, Grillon and Davis (1997) using an anticipation of electric shock procedure (that can be considered as the equivalent to psychological stress, since the participants never received the electric shock) found enhanced PPI in the stress condition as compared to a control "safe" condition that did not expect any shock, but similar PPI enhancement was found by merely indicating to the participants that they should actively attend to the different stimuli presented during the experiment. Therefore, the PPI increase can be attributed either to an emotional or to an attentional effect of the treatment (or a combination of both factors). Relatedly, when PPI has been registered in pathological populations diagnosed with anxiety disorders characterized by the presence of stress, such as Posttraumatic Stress Disorder (PTSD) or Panic Disorder (PD), PPI appeared disrupted both in medicated PD patients (Ludewig, Ludewig, Geyer, Hell, \& Vollenweider, 2002), and unmedicated PD patients (Ludewig, Geyer, Ramseier, Vollenweider, Rechsteiner, \& Cattapan-Ludewig, 2005). However, while several experiments have reported reduced PPI in PTSD patients (Grillon, Morgan, Davis, \& Southwick, 1998a, 1988b; Grillon, Morgan, Southwick, Davis, \& Charney, 1996; Ornitz \& Pynoos, 1989), others revealed intact PPI in similar 
populations (Butler, Braff, Rausch, Jenkins, Sprock, \& Geyer, 1990; Holstein, Vollenweider, Jäncke, Schopper, \& Csomor, 2010; Lipschitz, Mayes, Rasmusson, Anyan, Billingslea, Gueorguieva, \& Southwick, 2005).

The available evidence on emotional modulation of the startle amplitude is far more consistent. Thus, according to the emotional priming model proposed by Lang and his colleagues (Lang, 1995; Lang, Bradley, \& Cuthbert, 1990), the startle intensity is increased when it is elicited in the presence of aversive stimulation (e.g., Hawk, Stevenson, \& Cook, 1992; Ehrlichman, Brown, Zhu, \& Warrenburg, 1995; Vrana et al. 1988), but it is decreased when the stimuli are appetitive (e.g., Codispoti, Bradley, \& Lang, 2001; De la Casa et al., 2014; Sutton, Davidson, Donzella, Irwin, \& Dottl, 1997). Such emotional modulation of the startle response has been observed both in experiments with animals and with human participants, and is not dependent on the modality of the stimuli presented to induce the emotional state (Bradley, Cuthbert, \& Lang, 1999).

The main purpose of the Experiment 1 was to add evidence to the apparently contradictory results on the effects of stress on PPI. To this end, we registered the startle response in healthy participants who were submitted either to a stress condition by being engaged in a very difficult task (Stress Group), or to a very easy task (Control Group). Previous evidence evaluating the effect of stress on PPI makes it difficult to anticipate a result, but based on the results from rats and from PTSD patients, we expect a reduced PPI effect for those participants in the stress condition as compared to those participants in the control condition (Leitner, 1986; Grillon et al., 1996, 1998a). As for the effect of stress on startle magnitude, our hypothesis is clearer: we anticipate an enhanced startle reflex in the Stress Group as compared to the Control Group.

Since performing a difficult task requires a great amount of attentional resources, and some studies have demonstrated the effect of attentional manipulations on startle response and PPI (e.g., Blumenthal, 2001; Schicatano \& Blumenthal, 1998; Scholes \& Martin-Iverson, 2009; Thorne, Dawson, \& Schell, 2005), we also analyzed 
the possible effect of attentional demands on startle and PPI. More specifically, using an "attention-to-prepulse" paradigm that involves instructions to attend to one of two prepulses differing in pitch and duration while ignoring the other, it has been demonstrated that PPI was higher to the attended as compared to the non-attended prepulse (e.g., Ashare, Hawk, Mazzullo, 2007; Filion, Dawson, \& Schell, 1993). Therefore, the stress-mediated reduction of PPI we anticipated in Experiment 1 could be also related to reduced attention to the prepulse. To check this possibility, we employed in Experiment 2 the same parameters and stimuli from Experiment 1 to induce the startle response and PPI, but the participants were faced with a very simple task that required the allocation of a high amount of attentional resources (High Load [HL] Group) or to a task that did not require such effort (Low Load [LL] Group). If attention plays a role in PPI modulation in the first experiment, we would expect reduced PPI in the HL Group, but PPI should remain unchanged in the LL Group. As for the startle response, we expect a reduction of intensity in the $\mathrm{HL}$ as compared to the LL condition (e.g., Blumenthal, 2001)

\section{Experiment 1}

Stress corresponds to an emotional state that has been traditionally associated with relevant changes in learning and behavior, but it is a concept of difficult definition since it is composed of multiple components (Levine \& Ursin, 1991). From a physiological point of view, stress produces changes in the activity of the mesolimbic dopaminergic system (e.g., Funada \& Hara, 2001; Talalaenko, Abramets, Stakhovski, Shekhovtsov, Chernikov, \& Shevchenko, 1994), and in the opioid system (e.g., Van den Berg, Lamberts, Wolterink, Wiegant, \& Van Ree, 1998). Both physiological processes seem to be involved in startle and PPI modulation (Grillon \& Davis, 1997; Pijlman et al., 2003) that has favored the study of the relationship between stress, startle, and PPI (Grillon \& Davis, 1997; Grillon et al., 1996, 1998a, 1998b; Ellenbroek, van den Kroonenberg, \& Cools, 1998). 
A common definition of a stressful situation implies that the requirements or demands of such situations threaten or exceed the capacities of the individual (Lazarus \& Folkman, 1984). Accordingly, it is a common practice to induce situational stress in experimental situations by instructing the participants to solve intelligence-related tasks such as arithmetic tasks (e.g., Braunstein-Bercovitz, Dimentman-Ashkenazi, \& Lubow, 2001; Edwards, Moore, Champion, \& Edwards, 2015) or tests specifically designed to evaluate IQ as the Raven's progressive matrices test (e.g., Roskies, Seraganian, Oseasohn, Hanley, Collu, Martin, \& Smilga, 1986; Wrzesniewski, 1983). In our first experiment, we manipulated stress by differentially threatening participant's selfesteem by facing half of them (those in the Stress Group) to the most difficult items from the Advanced Progressive Matrices test (Raven, 1976). The remaining half of participants (those in the Control Group) were simply instructed to attend to a series of neutral images appearing on the computer screen, a common procedure used in our laboratory to minimize potential distractions in participants (e.g., De la Casa, Fernandez, Larrauri, Mena, Puentes, Quintero, \& Schmajuk, 2012). To induce startle and PPI the experimental treatment alternated trials involving presentations of an intense tone by itself (the Pulse, that allowed the startle reflex to be registered), and Prepulse-Pulse trials, consisting of the same intense tone preceded by a weaker sound.

\subsection{Method}

\subsubsection{Participants}

Twenty-two volunteers ( $n=11$ per group), 8 males and 14 females, participated in this experiment for course credits. Their ages ranged between 17 and 25 years. None of the participants reported any visual or hearing problem. All participants were informed of the type of stimulation used in the experiment, and provided signed informed consent before to start the experimental manipulations. Seville University's ethical committee approved the study. 


\subsubsection{Materials}

\subsubsection{Questionnaire.}

Levels of induced affect and arousal were assessed using the Mood Grid Scale (Russell, Weiss, \& Mendelson, 1989) that consists in a square divided in 81 cells organized in 9 rows and 9 columns, with the horizontal dimension representing emotion (from extremely unpleasant to extremely pleasant), and the vertical dimension representing arousal (from extremely low to extremely high). The responses to affect and arousal variables were transformed into scores ranging from 1 (minimum pleasantness/arousal) to 9 (maximum pleasantness/arousal).

\subsubsection{Inducing-stress task.}

The items 11-36 of the Set II from the Advanced Progressive Matrices test (Raven, 1976) were selected for the stress-inducing task. In order to increase the difficulty of the task, the items were presented in reverse order (starting with the more difficult ones). Each subject was instructed to identify the correct response for each item with a time limit of $15 \mathrm{~s}$. Those subjects in the Control condition were exposed to 26 neutral pictures selected from the International Affective Picture System (IAPS) and asked to identify the figure in a printed sheet containing a small reproduction of each picture. The mean IAPS valences for the images presented in the control condition were 5.34. Each image on both conditions was presented during $15 \mathrm{~s}$ without any temporal interval between them. Transition between images did not coincide with the occurrence of any auditory stimulus.

\subsubsection{Prepulse and pulse stimuli}

Acoustic stimuli were delivered binaurally using adjustable headphones (Sony model MDR-V50), connected to a MP150 control module (Biopac Systems Inc., Goleta, $\mathrm{CA})$. The signal was sent with a high sampling rate of $50 \mathrm{kHz}$. The prepulse and the pulse stimulus consisted of a $75 \mathrm{~dB}(\mathrm{~A})$ and $95 \mathrm{~dB}(\mathrm{~A})$ white noise with instantaneous rise time, lasting for 20 and $50 \mathrm{~ms}$, respectively. A background noise (white noise, 65 
$\mathrm{dB}$ ) was presented during the entire duration of the experiment. Sound calibration was completed prior to record data for each participant using a Sound Level Meter PCE999.

\subsection{Procedure}

The experiment was conducted in an isolated room. Before to start the experimental treatment, the participants were instructed to answer the Mood Grid Scale considering their actual affect and arousal, and then received instructions about the task they have to solve. Next, the headphones were put on and each participant was seated in front of a color monitor (approximately $100 \mathrm{~cm}$ from the eyes) controlled by a PC-computer were the items from the Raven test or the neutral images were presented. For all auditory trials, the ITI was $30 \mathrm{~s}(+/-5 \mathrm{~s})$ and the lead interval in prepulse-pulse trials was 40,60 or 80 ms After a $120 \mathrm{~s}$ adaptation period to the background noise, four pulses were presented in order to stabilize the response to the auditory stimuli. During this period, the computer's screen remained black. Then, the test stage consisting in 12 pulse-alone and 12 prepulse-pulse alternated trial presentations was initiated ( 3 trials for each lead interval, presented in a random order across the experimental session). The corresponding task for the Stress and Control groups was presented simultaneously to the auditory stimuli presentation. Finally, the earphones were removed, and each participant was asked again to answer the Mood Grid Scale considering the affective state perceived during the experimental stage.

Electromyographic (EMG) activity of the orbicularis oculi muscle was recorded using three $\mathrm{Ag} / \mathrm{AgCl}$ electrodes (EL250; Biopac Systems) positioned according to the guidelines recommended by Blumenthal, Cuthbert, Filion, Hackley, Lipp and Van Boxtel (2005). Specifically, after cleaning the participant's skin, conductive gel was applied to the electrodes before placing two of them approximately $1 \mathrm{~cm}$ below the right eye to record the electromyographic activity of the orbicularis oculi muscle. The third electrode was placed on the forehead to detect the general level of electrical activity. Raw signals were amplified (×2000) and filtered using a passband of $10-500$ 
Hz (EMG100C amplifier; Biopac Systems). AcqKnowledge software (4.0, Biopac Systems) was used to interface a MP150 control module (Biopac Systems) via a crossover cable and sampled at $2 \mathrm{kHz}$. Response onset latency windows include 21- 120 ms for acoustically elicited blinks.

\subsection{Results.}

\subsubsection{Analyses of Mood Grid scores}

Mean scores from the Mood Grid for Arousal and Affect as a function of stage (pre- and post-experimental) appear in the upper section of Table 1. As can be seen in the Table, the Arousal scores increased after the experimental treatment for the Stress Group, but remained unchanged for the Control Group. As for the affect scores, there was a decrease after the experimental treatment that was more intense for the Stress Group.

Table 1 about here

These impressions were confirmed by the statistical analyses: A $2 \times 2$ mixed ANOVA (Stage: Pre vs. Post x Group: Stress vs. Control) conducted on mean arousal scores obtained in the Mood Grid revealed a significant main effect of Stage, and a significant Stage $\times$ Group interaction, $\underline{F}(1,20)=4.42 ; \underline{p}<.05, \eta_{p}^{2}=.18$, and $\underline{F}(1,20)=5.52$; $\underline{p}<.05, \eta_{\mathrm{p}}^{2}=.22$, respectively. The main effect of Groups was non-significant, $\underline{F}(1,20)=2.67 ; \underline{p}>.11, \eta_{p}^{2}=.12$. The 2 -way interaction was explored by comparing preand post-experimental scores for each group (t.-test for related samples, one-tailed, $\underline{\mathrm{p}}<.05)$. The comparisons revealed a significant increase from pre- to post-experimental stage in arousal scores that was restricted to the Stress Group. This result indicates that the manipulation introduced to induce stress produced an increase in arousal the participants.

A similar analysis conducted on mean affect scores revealed a significant main effect of Stage, and a significant Stage $\times$ Group interaction, $\underline{F}(1,20)=36.03 ; \underline{p}<.001, \eta_{p}^{2}$ 
$=.64$, and $\underline{F}(1,20)=12.42 ; \underline{p}<.01, \eta_{p}{ }^{2}=.35$, respectively. The main effect of Groups was close to the standard levels of significance, $\underline{F}(1,20)=4.33 ; \underline{p}=.051, \eta_{p}^{2}=.18$. The Stage x Group interaction was explored by comparing pre- and post-experimental scores for each group (t-test for related samples, one-tailed, $\underline{\mathrm{p}}<.05$ ) that revealed a significant reduction from pre- to post-experimental stage in affect scores but only for the Stress Group. This result seems to indicate that the manipulation introduced to induce stress produced a negative affect in the participants.

\subsubsection{Analysis of the startle response to the Pulse-alone trials}

A preliminary analysis was conducted on mean startle to the four pulses included to stabilize the responses to the Pulse that were presented before to start the experimental treatment. This analysis was intended to identify possible differences in startle reactivity between groups that could have affected to PPI magnitude. Specifically, a 4 × 2 mixed ANOVA (Trials x Group: Stress vs. Control) revealed a significant main effect of Trials, $\underline{E}(3,60)=5.76 ; \underline{p}<.01, \eta_{p}^{2}=.22$, reflecting the habituation of the startle response across trials. Neither the main effect of Group nor the 2-way interaction was significant, both ps>.27.

Figure 1 depicts mean pre-test startle magnitude (averaged across trials), and mean startle magnitude for the 12 Pulse-alone experimental trials as a function of Groups. As can be seen in the figure, there was a general reduction in the startle response across trials that reflect the habituation process. Also, the startle response magnitude was lower for the participants in the stress condition as reflected by the immediate drop in startle reactivity that was evident from the first test trial for the Stress Group.

Figure 1 about here 
These impressions were confirmed by a $12 \times 2$ mixed ANOVA (Trials $\times$ Group: Stress vs. Control) conducted on mean startle intensity to the Pulse-alone trials that revealed significant main effects of Trials, $\underline{F}(19,220)=2.26 ; \underline{p}<.05, \eta_{p}{ }^{2}=.10$, reflecting the overall habituation of the startle to the Pulse. The main effect of Groups was also significant, $\underline{F}(1,20)=6.65 ; \underline{p}<.05, \eta_{p}^{2}=.25$, due to an overall lower mean startle response in the Stress as compared to the Control Group (Mean $=.36 \mu \mathrm{V}, \mathrm{SD}=.14$, and Mean $=.64 \mu \mathrm{V}, \mathrm{SD}=.34$, respectively). This result was unexpected, since it has been consistently reported that the induction of an unpleasant emotional state results in an increase of the startle response to an acoustic stimulus (see, for a review, Grillon \& Baas, 2003). Finally, the 2-way interaction was non-significant, $\underline{F}(11,220)=1.47$; p $>.14$.

\subsubsection{Analyses of percent PPI}

Since several studies have suggested that percent PPI is less contaminated by individual differences than raw PPI (e.g., Hawk \& Cook, 2000; Schwarzkopf, McCoy, Smith, \& Boutros, 1993), mean startle magnitudes for pulse and prepulse-pulse trials were converted into percent PPI, calculated as $100 \times$ ([Average startle to the pulse Average startle to the prepulse-pulse]/ Average startle to the pulse). Figure 2 shows mean PPI percent collapsed across trials for each lead interval condition as a function of groups. As can be seen in the Figure, PPI was reduced in the Stress as compared to the Control Group in the $60 \mathrm{~ms}$ and $80 \mathrm{~ms}$ lead conditions.

Figure 2 about here

These impressions were confirmed by the statistical analyses, since a $2 \times 3$ mixed ANOVA (Group: Stress vs. Control x Lead interval: 40 vs. 60 vs. 80 ms) conducted on mean percent PPI revealed a significant main effect of Group, $\underline{F}(1,20)=5.43 ; \underline{p}<.05, \eta_{p}^{2}=.21$, and a significant Group $x$ Lead interval interaction, $\underline{F}(2,40)=4.90 ; \underline{p}<.05, \eta_{p}^{2}=.20$. The main effect of Lead felt short of significance, 
$\underline{F}(2,40)=2.62 ; \underline{p}=.085, \eta_{p}^{2}=.12$. In order to explore the source of the 2-way interaction, we conducted independent one-way ANOVAs for each Lead condition (40 ms vs. 60 ms vs. $80 \mathrm{~ms}$ ) on mean percent PPI with Group as main factor. The ANOVA for the 40 ms lead interval condition was non-significant, $\underline{F}(1,20)<1$. However, there were significant differences for the 60 and 80 ms conditions, $\underline{F}(1,20)=6.35 ; \underline{p}<.05, \eta_{p}^{2}=.24$, and $\underline{F}(1,20)=7.15 ; \underline{p}<.05, \eta_{p}^{2}=.26$, respectively, indicating that $P P I$ was reduced in the Stress as compared to the Control Group.

In summary, the results indicate that there was a different effect of inducedstress on PPI as a function of the lead interval between Pulse and Prepulse-Pulse. Thus, with the shorter interval (40 ms) the PPI was very weak in the Control Group that probably resulted in a floor effect that impedes to detect possible differences between groups. However, the PPI effect was higher in the control Group when the lead interval was 60 and $80 \mathrm{~ms}$, giving thus the opportunity to modulate the startle intensity as a function of the psychological effect induced by the task. As predicted, PPI was reduced when the participants were confronted to the stress-inducing task in the 60 and $80 \mathrm{~ms}$ lead conditions.

\section{Experiment 2}

The reduced PPI obtained in the Stress Group in Experiment 1 reproduces the results reported in previous research with rats, and with PTSD and PD patients (e.g., Grillon et al., 1996; Ludewig et al., 2005; Pijlman et al., 2003). However, the presence of a high attentional load task intended to induce stress during the auditory stimuli presentation in our experiment could have introduced a confounding factor, since PPI is sensitive to attentional manipulations. In fact, it has been reported that increasing attention to the Prepulse stimulus results in an enhancement of PPI as compared to an unattended condition (e.g., Filion \& Poje, 2003; Thorne et al., 2005). Therefore, we can consider that performing a highly demanding task during acoustic stimuli presentation, as we programmed in Experiment 1, could have resulted in a reduction of the attention 
paid to the Prepulse, and that this reduced attention could have contributed to the reduced PPI reported in Experiment 1. Similarly, the reduced startle response observed for the Stress as compared to the Control Group in Experiment 1 can be interpreted as the result of diverting the participants' attention away from the auditory stimuli (see, for a similar result, Schicatano \& Blumenthal, 1998).

To check the possible effect of the attentional process on PPI modulation, we conducted an additional experiment with the same parameters to induce PPI as described in Experiment 1, but in this case the participants were instructed to perform one of two simple tasks (neither of them related to intelligence) that differed in attentional demands (High attentional load vs. Low attentional load). Those participants in the High Load (HL) Group were instructed to respond to the Toulouse-Piéron perceptive and attentional test (Toulouse \& Piéron, 1986), a test specifically designed to evaluate attentional resources. It is considered as a test that required a big amount of concentration, since it is composed by 1600 shapes (small squares each one with a script in one of the sides or angles) all presented in one single sheet. The required response is to detect and cross out with a pencil those shapes similar to anyone of the two models reproduced on the top of the response sheet (there are 400 shapes similar to the models randomly distributed). As for the Low Load (LL) Group, the participants were instructed to pay attention to the computer screen were the same 26 neutral pictures described for Experiment 1 were presented (15 sec. each). In order to make this task more similar to that presented for the HL Group, the participants were instructed to locate each image in a printed sheet containing a small reproduction of each picture. Whether the reduced PPI observed in Experiment 1 was the result of an attentional process, we would expect a similar effect in the High load Group. Similarly, if the reduction of the startle magnitude observed in the Stress Group in Experiment 1 was due to the limited attentional resources available while performing the difficult task, we expect a similar reduced startle in the High load as compared to the Control Group.

\subsection{Method}




\subsubsection{Participants}

Twenty-two volunteers ( $n=11$ per group), 3 males and 19 females, participated in this experiment for course credits. Their ages ranged between 18 and 25 years. None of the participants reported any visual or hearing problem. As in Experiment 1, the participants were informed of the type of stimulation used in the experiment, and provided signed informed consent before to start the experimental manipulations. Seville University's ethical committee approved the study.

\subsubsection{Materials}

\subsubsection{Questionnaire}

The questionnaire employed to evaluate arousal and affect was the same as described for Experiment 1 (Mood Grid).

\subsubsection{Attentional-related tasks:}

In this experiment the participants were divided in two groups. Those in the $\mathrm{HL}$ Group were instructed to solve the Toulouse-Piéron perceptive and attentional test (Toulouse \& Piéron, 1986). Those participants assigned to the LL Group were exposed to the same 26 neutral pictures described in Experiment 1, and instructed to locate and cross out with a pencil each picture on a sheet that contained a small reproduction of all 26 images.

\subsubsection{Prepulse and pulse stimuli}

All acoustic stimuli and temporal parameters were the same as described for Experiment 1.

\subsection{Procedure}

Before to start the experiment, the participants were instructed about the task they have to complete. Thus, the participants in the HL Group received the instructions to complete the Toulouse-Piéron test, and those in the LL Group were instructed to attend to the different images that will appear on the computer screen, and to identify the matching image in a printed sheet. For both experimental groups the screen 
computer remained black during the pre- and post-experimental stages. When the experimental stage started the phrase "start the test" appeared on the screen for the HL Group, while for the LL Group the presentation of the visual stimuli (the neutral images from the IAPS) started. The remaining procedural details were exactly as described for Experiment 1.

Physiological data collection was similar to that described for Experiment 1.

\subsection{Results.}

\subsubsection{Analyses of Mood Grid scores.}

Mean scores in the Mood Grid for Arousal and Affect as a function of stage (pre- and post-experimental) appears in the lower section of Table 1. Two similar $2 \times 2$ mixed ANOVA (Stage: Pre vs. Post x Group: LL vs. HL) conducted on mean arousal and affect scores revealed no significant main effects or interactions (all ps $>.09$ ). Therefore, and conversely to that observed in Experiment 1, the tasks in this experiment did not induce neither arousal nor affective changes in the participants.

\subsubsection{Analysis of startle to the Pulse-alone trials}

As in Experiment 1, we conducted a preliminary analysis on mean startle to the four pulses presented before the experimental treatment. A $4 \times 2$ mixed ANOVA (Trials $x$ Group: LL vs. $H L)$ revealed a significant main effect of Trials, $\underline{F}(3,60)=3.10 ; \underline{p}<.05, \eta_{p}{ }^{2}$ $=.13$, reflecting the habituation of the startle response across trials. Neither the main effect of Group nor the 2-way interaction was significant, both ps $>.38$.

In order to identify a possible effect of the treatment on startle to the Pulse, we analyzed mean startle responses to the Pulse-alone trials presented during the experimental phase. As can be seen in Figure 3, that depicts mean pre-test startle intensity (collapsed across trials), and mean startle magnitude to the Pulse-alone trials as a function of Groups, it appeared a general decrease of startle magnitude across trials due to a habituation process, that was more evident for the LL Group. In addition, the startle magnitude was lower for the $\mathrm{HL}$ than for the LL Group. 
Figure 3 about here

These impressions were confirmed by a $12 \times 2$ mixed ANOVA (Trials $\times$ Groups: LL vs. HL) conducted on mean startle magnitude to the experimental Pulse-alone trials, that revealed significant main effects of Trials and Groups, $\underline{F}(11,220)=2.89 ; \underline{p}<.01, \eta_{p}{ }^{2}$ $=.13$, and $\underline{F}(1,20)=12.31 ; \underline{\mathrm{p}}<.01, \mathrm{\eta}_{\mathrm{p}}{ }^{2}=.38$, respectively. The 2-way interaction was also significant, $\underline{F}(11,220)=2.19 ; \underline{p}<.05, \eta_{p}^{2}=.10$. As can be seen in Figure 3, the interaction reflects that the habituation of the startle response was restricted to the LL Group.

\subsubsection{Analyses of percent PPI}

As in Experiment 1, mean startle magnitudes for pulse and prepulse-pulse trials were transformed into percent PPI. Figure 4 shows mean PPI percent collapsed across trials for each lead interval condition as a function of Groups. As can be seen in the Figure, PPI appeared for all lead conditions, with higher PPI levels for the 60 and 80 ms lead interval conditions, but there were no differences in PPI of startle intensity between groups.

Figure 4 about here

These impressions were confirmed by the statistical analyses. A $3 \times 2$ mixed ANOVA (Lead interval: 40 vs. 60 vs. 80 ms x Group: LL vs. HL) conducted on mean percent PPI revealed only a significant main effect of Lead interval, $\underline{F}(2,40)=6.66$;

$\underline{\mathrm{p}}<.01, \mathrm{\eta}_{\mathrm{p}}^{2}=.25$ (the remaining $\underline{\mathrm{p}} \mathrm{s}>.79$ ). Pairwise-comparisons (t-tests for independent samples, $\underline{\mathrm{p}}<.05$, one-tailed) conducted on percent $\mathrm{PPI}$ revealed that mean percent PPI was weaker for the 40 ms condition (Mean $=13.06 \%, S D=19.06$ ) as compared to the $60 \mathrm{~ms}$, and $80 \mathrm{~ms}$ condition $($ Mean $=24.36 \%, \mathrm{SD}=24.87$, and Mean $=29.04 \%, \mathrm{SD}=$ 21.09 , respectively). 
In summary, the results indicate that mean startle response magnitude was reduced when the participants were confronted to the high attentional demanding task. However, there was no effect of the attentional manipulation on PPI intensity.

\section{General Discussion}

The present study analyzed the effect of stress and attention on startle intensity and PPI. As predicted, the introduction of a very difficult task for the Stress Group reduced PPI intensity as compared to the Control Group in Experiment 1. However, and contrary to our expectations, the startle response intensity was reduced in the Stress Group as compared to the Control Group, that we interpreted as the result of diverting the attention away from the startling stimulus. In fact, the decrease of startle was too sudden and stable to be due to accumulation of stress in the early trials, and is attributable to the requirements of the task.

Since the reduced PPI observed in Experiment 1 could be the result of the same attentional process that disrupted startle intensity, Experiment 2 was designed to check for a possible effect of attentional factors on PPI disruption. Specifically, we created two groups differing in the amount of attention the participants had to invest in a task that they had to solve while registering startle intensity and PPI. Both high- and low-load tasks were selected according to their easiness, and anticipating that they would not induce stress in the participants (in fact, affect and arousal measures remained unchanged in both groups in Experiment 2 as revealed by a comparison of the pre- and post-experimental scores from the mood grid scale). The differential effect of attentional load was evident when we analyzed the startle intensity in the Pulsealone trials, since it appeared significantly reduced in the High load Group as compared to the Low load Group. This result confirmed the disrupting effect of diverting attention away from the auditory stimuli on the startle response observed in Experiment 1 (for similar results see, Anthony \& Graham, 1985; Hackley \& Graham, 1983; 
Hutchison, McGeary, Wooden, Blumenthal, \& Ito, 2003; Schicatano \& Blumenthal, 1998). However, PPI intensity remained unchanged in Experiment 2 in spite of the attentional manipulation, which seems to indicate that the reduced PPI observed in the Stress Group from Experiment 1 was produced by the emotional changes induced by the extremely difficult task programmed for this group.

\subsection{Startle reflex, PPI, and stress.}

Regarding the effects of emotional changes on startle response and PPI, there are two relevant lines of research in the literature with human participants that have analyzed: i) the effect of positive and negative induced-affect on startle response intensity (e.g., Bradley \& Lang, 2000; Lang et al., 1990; Grillon \& Baas, 2003) and PPI (e.g., De la Casa et al., 2014; Hawk \& Cook, 2000; Vanman, Boehmelt, Dawson, \& Schell, 1996; Sommer, Van der Molen, \& Pascalis, 2016), and ii) the effect of high anxiety levels or stress on startle intensity (e.g., Grillon, Dunco, Covington, Copperman, \& Kling, 2007) and PPI both with healthy (Grillon \& Davis, 1997) and pathological populations (e.g., Grillon et al., 1996, 1998a).

More specifically, the evidence on startle magnitude modulation by both induced-affect and stress is quite consistent (e.g., Bradley et al., 1999; Grillon \& Baas, 2003), since startle is increased in the presence of unpleasant stimuli (e.g., Vrana et al., 1988) or after stress treatment (e.g., Grillon et al., 2007), and it is reduced when registered during pleasant stimuli presentation (e.g., De la Casa et al., 2014; Vrana et al., 1988). Lang et al. (1990) have proposed that startle modulation by induced-affect is the result of a motivational priming effect that depends on a comparison between the current affective state of the individual and the valence of the reflex, in such way that when the reflex valence (appetitive or aversive) matched the current affective state (positive or negative), the reflex magnitude will increase. Conversely, a mismatch between the reflex and the affective state will result in response attenuation. 
However, the results regarding PPI modulation by induced affect or stress are far more inconclusive. On the one hand, the available evidence has revealed either intact PPI in spite of the induction of different affects (e.g., Hawk \& Kowmas, 2003; Sommer et al., 2016), or reduced PPI when the induced affect is negative (e.g., Vanman et al., 1996), or even increased PPI with positive-induced affect (De la Casa et al., 2014). On the other hand, research evaluating the effects of stress on PPI revealed enhanced PPI in an experiment with human participants and a threat-of-shock procedure (Grillon \& Davis, 1997), but PPI appeared disrupted in children with PTSD (Ornitz \& Pynoos, 1989), and in Vietnam veterans with PTSD (Grillon et al., 1996, 1998a). Similarly, disrupted PPI has been found in medicated and unmedicated PD patients (Ludewig et al., 2002, 2005). Finally, there is also experimental evidence showing intact PPI in PTSD patients (Butler et al., 1990; Grillon et al., 1998b; Holstein et al., 2010).

\subsection{Startle, PPI, and attention.}

The effects of attention on acoustic startle reflex modulation have been considered from both the psychological (e.g., Lang et al., 1990) and the physiological (e.g., Bohlin, Graham, Silverstein, \& Hackley, 1981) perspectives. The experimental evidence shows that startle increases when the attention is directed to the startle stimulus (e.g., Anthony \& Graham, 1985; Blumenthal, 2001), and that it decreases when attention is directed away from the startle stimulus (e.g., Schicatano \& Blumenthal, 1998). The reduced startle response observed in the experimental groups from Experiment 1 and 2 (Stress Group, and High load Group, respectively) is consistent with the aforementioned literature, and seems to confirm that attentional resources are limited, in such way that when attention is engaged in a stimulus of a specific modality (e.g., solving a visual task), the resources required to attend to stimuli of a different modality are diminished (e.g., the auditory stimuli). 
Regarding the effects of attentional processes on PPI, early theories proposed that processing of the Prepulse stimulus triggered an automatic preattentive process that resulted in a reduced response to the more intense pulse (Graham, 1975). However, more recent hypotheses have considered PPI as a sensory-motor gating of startle that is related to attentional processes (e.g., Filion et al., 1993). Thus, for instance, Thorne et al. (2005) conducted an experiment analyzing the role of attention on PPI, and concluded that an increase in attention to the prepulse stimulus resulted in PPI enhancement. Usually, in those experiments designed to evaluate the impact of attention on PPI, the participant's attention is directed to the Prepulse trials by instructing them, for instance, to identify the length or intensity of the stimuli, and the resulting startle response is compared to that produced by alternating non-attended trials without any instruction regarding the stimuli (e.g. Filion \& Poje, 2003; Jennings, Schell, Filion, \& Dawson, 1996). In general, such experimental manipulations have resulted in a PPI enhancement for the attended as compared to the non-attended trials that has been interpreted as the result of an increase in the protection of the information mechanism responsible for the PPI effect (Thorne et al., 2005)

\subsection{Stress, dopamine and PPI.}

The reduced PPI induced by stress reported in previous research and in our Experiment 1, can be explained by the elevated dopaminergic activity that follows stress induction (e.g., Salamone, Cousins, \& Snyder, 1997; Talalaenko et al., 1994), and by consideration of the relationship between PPI and dopaminergic activity (e.g., Schmajuk et al., 2009; Zhang, Forkstam, Engel, \& Svensson, 2000). Thus, it is well established that dopamine plays a crucial role in modulating PPI since dopamine agonists administration reduces PPI intensity (e.g., Swerdlow \& Geyer, 1998, Swerdlow, Stephany, Talledo, Light, Braff, Baeyens, \& Auerbach, 2005), and dopamine antagonists facilitate PPI expression (e.g., Swerdlow, Keith, Braff, \& Geyer, 1991). 
Converging evidence shows that the induction of stress increases levels of dopamine in the nucleus accumbens (e.g., Tidey \& Miczek, 1996).

Schmajuk and Larrauri (2005) proposed a neurobiological circuit responsible for mediating and regulating acustic startle responses and PPI. The model that integrates previous proposals by Koch (1999) and Swerdlow and Geyer (1999), is composed of an excitatory and an inhibitory pathway. The excitatory pathway includes the Cochlear Nucleus $(\mathrm{CN})$ which projects to the Caudal Pontine reticular nucleus $(\mathrm{PnC})$ that activates the spinal motor neurons responsible for the startle response. The inhibitory pathway includes the $\mathrm{CN}$, the Inferior Colliculus (IC), and the Pedunculopontine Tegmental Nucleus (PPT) that inhibits PnC and produces PPI. The model also incorporates different brain areas that regulate the circuit responsible for PPI: the Ventral Tegmental Area (VTA), the nucleus accumbens (NAc), and the ventral pallidum (VP). According to the model, variations in phasic dopaminergic activity from the VTA to NAc, modulate activity in the PPT through direct NAc-PPT and indirect NAc-VP-PPT GABAergic inhibitory projections. Albeit speculative, considering that there is evidence of dopamine release in the NAc during social threat (Tidey \& Miczek, 1996) and acute restraint stress (Anstrom \& Woodward, 2005), we can apply the model to our results hypothesizing that stress was responsible for PPT inhibition. As a result, the excitatory input from the IC to the PPT activated by the prepulses was less effective in reducing PPI.

\section{Conclusion.}

We studied the effects of stress and attention on the startle response and PPI. Our results seem to indicate that induced stress can reduce PPI through midbrain dopamine activity, and that startle reflex intensity is reduced when the attention is directed away from the auditory stimulus that induces the reflex. Therefore, both the startle reflex and PPI can be considered as useful tools to study the effects of emotional and attentional processes in healthy and pathological populations. 
STRESS, STARTLE AND PPI 24 


\section{Acknowledgments.}

This research was supported by grants from Spanish Ministerio de Economia y Competitividad (PSI2012-32077, and PSI2015-64965-P/MINECO-FEDER, UE). The authors wish to thank Juan Carlos Lopez for helpful comments on an early version of this manuscript, and Inmaculada Ruiz for her help in running the experiments.

Correspondence concerning to this article should be addressed to L. Gonzalo De la Casa (delacasa@us.es), Departamento de Psicología Experimental, C/ Camilo Jose Cela, s/n. 41018 Sevilla (Spain). 


\section{References.}

Anstrom, K.K., \& Woodward, D.J. (2005). Restraint increases dopaminergic burst firing in awake rats. Neuropsychopharmacology, 30, 1832-1840.

Anthony, B. J., \& Graham, F. K. (1985). Blink reflex modification by selective attention, Evidence for the modulation of 'automatic' processing. Biological Psychology, $\underline{21}, 43-59$.

Ashare, R.L., Hawk Jr., L.W., \& Mazzullo, R.J. (2007). Motivated attention: incentive effects on attentional modification of prepulse inhibition. Psychophysiology $\underline{44}, 839-845$.

Blumenthal, T. D. (2001). Extraversion, attention, and startle response reactivity. Personality and Individual Differences, 30, 495-503.

Blumenthal, T.D. (2015). Presidential Address 2014: The more-or-less interrupting effects of the startle response. Psychophysiology, 52, 1417-1431.

Blumenthal, T. D., Cuthbert, B. N., Filion, D. L., Lipp, O. V., van Boxtel, A., \& Hackley, S. (2005). Committee report, Guidelines for human startle eyeblink electromyographic studies. Psychophysiology, 42, 1-15.

Bohlin, G., Graham, F. K., Silverstein, L. D., \& Hackley, S. A. (1981). Cardiac orienting and startle blink modulation in novel and signal situations. Psychophysiology, 18, 603-611.

Bradley, M.M., Cuthbert, B.N., \& Lang, P.J. (1999). Affect and the Startle Reflex. In, M.E. Dawson, A.M. Schell, \& A.H. Boehmelt (Eds.), Startle Modification, Implications for Neuroscience. Cognitive Science and Clinical Science (pp. 157-183). New York: Cambridge University Press.

Bradley, M.M., \& Lang, P.J. (2000). Measuring emotion, Behavior, feeling, and physiology. In, R.D. Lane, \& L. Nadel (Eds.), Cognitive neuroscience of emotion (pp. 242-276). New York: Oxford University Press.

Braff, D. L., Geyer, M. A., \& Swerdlow, N. R. (2001). Human studies of prepulse inhibition of startle, Normal subjects, patient groups, and pharmacological studies. Psychopharmacology, 156, 234-258.

Braff, D.L., Grillon, C., \& Geyer, M.A. (1992). Gating and habituation of the startle reflex in schizophrenic patients. Archives General Psychiatry, 49, 206-215. 
Braunstein-Bercovitz H., Dimentman-Ashkenazi, I., Lubow, R. E. (2001). Stress Affects the Selection of Relevant From Irrelevant Stimuli. Emotion, 1, 182-192.

Butler, R.W., Braff, D.L., Rausch, J.L., Jenkins, M.A., Sprock, J., \& Geyer, M.A. (1990). Physiological evidence of exaggerated startle response in a subgroup of Vietnam veterans with combat-related PTSD. The American Journal of Psychiatry, 147, 1308-1312.

Codispoti, M., Bradley, M.M., \& Lang, P.J. (2001). Affective reactions to briefly presented pictures. Psychophysiology, 38, 474-478.

Dahmen, J.C., \& Corr, P.J. (2004). Prepulse-elicited startle in prepulse inhibition. Biological Psychiatry, 55, 98-101.

Dawson, M.E., Schell, A.M., \& Böhmelt, A.H. (1999). Startle Modification, Introduction and Overview. In M.E. Dawson, A.M. Schell, \& A.H. Boehmelt (Eds.), Startle Modification, Implications for Neuroscience. Cognitive Science and Clinical Science (pp. 257-275). New York: Cambridge University Press.

De la Casa, L. G., Mena, A., \& Puentes, A. (2014). Startle response and prepulse inhibition modulation by positive- and negative-induced affect. International Journal of Psychophysiology, 91, 73-79.

De la Casa, L.G., Fernandez, A., Larrauri, J., Mena, A., Puentes, A., Quintero, E., \& Schmajuk, N. (2012). Different effects of unexpected changes in environmental conditions on prepulse inhibition in rats and humans, Physiology \& Behavior, 106, 542 547.

Edwards, M.S., Moore, P., Champion, J.C., and Edwards, E.J. (2015). Effects of trait anxiety and situational stress on attentional shifting are buffered by working memory capacity. Anxiety, Stress \& Coping: An International Journal, 28,1-16

Ehrlichman H., Brown S., Zhu J., \& Warrenburg S. (1995). Startle reflex modulation during exposure to pleasant and unpleasant odors. Psychophysiology, 32, 150-154.

Ellenbroek, B.A., van den Kroonenberg, P.T.J.M, \& Cools, A.R. (1998). The effects of an early stressful life event on sensorimotor gating in adult rats.

Schizophrenia Research, 30, 251-260.

Filion, D. L., Dawson, M. E., \& Schell, A. M. (1993). Modification of the acoustic startle-reflex eyeblink, $A$ tool for investigating early and late attentional processes.

Biological Psychology, 35, 185-200. 
Filion, D.L., \& Poje, A.B. (2003). Selective and nonselective attention effects on prepulse inhibition of startle, A comparison of task and no-task protocols. Biological Psychology, 64, 283-296.

Funada, M., \& Hara, C. (2001). Differential effects of psychological stress on activation of the 5-hydroxytryptamine- and dopamine-containing neurons in the brain of freely moving rats. Brain Research, 901, 247-251.

Graham, F.K. (1975). The more or less startling effects of weak pre-stimulation. Psychophysiology, 12, 238-248.

Grillon, C.,, \& Baas, J. (2003). A review of the modulation of the startle reflex by affective states and its application in psychiatry. Clinical Neurophysiology, 114, 15571579.

Grillon, C., \& Davis, M. (1997). Effects of stress and shock anticipation on prepulse inhibition of the startle reflex. Psychophysiology, 34, 511-517.

Grillon, C., Duncko, R., Covington, M.F., Kopperman, L., \& Kling, M.A. (2007) Acute Stress Potentiates Anxiety in Humans. Biological Psychiatry, 62, 1183-1186.

Grillon, C. Morgan , C. A. , Southwick, S. M., Davis , M., \& Charney , D. S. (1996). Baseline startle amplitude and prepulse inhibition in Vietnam veterans with posttraumatic stress disorder. Psychiatry Research, 64, 169-178.

Grillon, C., Morgan, C.A., Davis, M., \& Southwick, S.M. (1998a). Effects of experimental context and explicit threat cues on acoustic startle in Vietnams veterans with prosttraumatic stress disorder. Biological Psychiatry, 44, 1027-1036.

Grillon, C., Morgan, C.A., Davis, M., \& Southwick, S.M. (1998b). Effect of darkness on acoustic startle in Vietnam veterans with PTSD. The American Journal of Psychiatry, 155, 812-817.

Gottesman, I.I., \& Gould, T.D. (2003) The endophenotype concept in psychiatry, etymology and strategic intentions. The American Journal of Psychiatry, 160, 636-645.

Hackley, S. A., \& Graham, F. K. (1983). Early selective attention effects on cutaneous and acoustic blink reflexes. Physiological Psychology, 11, 235- 242.

Hawk, L.W., Cook, E.W. (2000). Independence of valence modulation and prepulse inhibition of startle. Psychophysiology, 37, 5-12.

Hawk, L.W., \& Kowmas, A.D. (2003). Affective modulation and prepulse inhibition of startle among undergraduates high and low in behavioural inhibition and approach. Psychophysiology, 40, 131-138. 
Hawk, L.W., Stevenson, V.E., \& Cook, E.W. (1992). The effects of eyelid closure on affective imagery and eyeblink startle. Journal of Psychophysiology, 6, 299310.

Hoenig, K., Hochrein, A., Quednow, B.B., Maier, W., \& Wagner, M. (2005). Impaired prepulse inhibition of acoustic startle in obsessive-compulsive disorder. Biological Psychiatry, 57, 1153-1158.

Hoffman, H.S., \& Searle, J. (1968). Acoustic and temporal factors in the evocation of startle. The Journal of the Acoustical Society of America, 43, 269-282.

Holstein, D.H., Vollenweider, F.X., Jancke, L., Schopper, C., \& Csomor, P.A. (2010). P50 suppression, prepulse inhibition, and startle reactivity in the same patient cohort suffering from posttraumatic stress disorder. Journal of Affective Disorders, 126, 188-197.

Huppert, J.D., Weiss, K.A., Lim, R., Pratt, S., \& Smith, T.E. (2001). Quality of life in schizophrenia, contributions of anxiety and depression. Schizophrenia Research, 51, 171-80.

Hutchinson, K. E., McGeary, J., Wooden, A., Blumenthal, T. D., \& Ito, T. (2003). Startle magnitude and prepulse inhibition, Effects of alcohol and attention.

Psychopharmacology, 167, 235-241.

Jennings, P. D., Schell, A. M., Filion, D. L., \& Dawson, M. E. (1996). Tracking early and late stages of information processing, Contributions of startle blink reflex modification. Psychophysiology, 33, 148-155.

Koch, M. (1999). The neurobiology of startle. Progress in Neurobiology, 59, $107-128$.

Kohl, S., Heekerenb, K., Klosterköttera, J., \& Kuhn, J. (2013). Prepulse inhibition in psychiatric disorders - apart from schizophrenia. Journal of Psychiatric Research, 47, 445-452.

Lang, P.J. (1995). The emotion probe, studies of motivation and attention. The American Psychologist, 50, 372-385.

Lang, P., Bradley, M., \& Cuthbert, B. (1990). Emotion, attention and the startle reflex. Psychological Review, 97, 377-395.

Larrauri, J., \& Schmajuk, N. (2006). Prepulse inhibition mechanisms and cognitive processes. A review and model. In E.D. Levin (Ed.), Neurotransmitter 
Interactions and Cognitive Function (pp. 245-278). Basel, Switzerland: Birkhauser Verlag.

Lazarus, R. S., \& Folkman, S. (1984). Stress, appraisal, and coping. New York: Springer.

Leitner, D.S. (1986) Alterations in other sensory modalities accompanying stress analgesia as measured by startle reflex modification. Annals of the New York Academy of Science, 467, 82-92.

Levine, S., \& Ursin, H. (1991) What is stress? In, M.R. Brown, \& G.F. Koob (Eds.), Stress Neurobiology and Neuroendocrinology (pp. 3-21). New york: Rivier Marcel Dekker.

Light, G.A., Swerdlow, N.R., Rissling, A.J., Radant, A., Sugar, C.A., Sprock, J., Pela, M., Geyer, M.A., \& Braff, D.L. (2012). Characterization of neurophysiologic and neurocognitive biomarkers for use in genomic and clinical outcome studies of schizophrenia. PLoS One, 7, e39434.

Lipschitz, D.S., Mayes, L.M., Rasmusson, A.M., Anyan, W., Billingslea, E., Gueorguieva, R., \& Southwick, S.M. (2005). Baseline and modulated acoustic startle responses in adolescent girls with posttraumatic stress disorder. Journal of the American Academy of Child and Adolescent Psychiatry, 44, 807-814.

Ludewig, S., Geyer, M.A., Ramseier, M., Vollenweider, F.X., Rechsteiner, E., \& Cattapan-Ludewig, K. (2005). Information processing deficits and cognitive dysfunctions in panic disorder. Journal of Psychiatry, \& Neuroscience, 30, 37-43.

Ludewig, S., Ludewig, K., Geyer M.A., Hell, D., \& Vollenweider, F.X. (2002). Prepulse inhibition deficits in patients with panic disorder. Depression and Anxiety, 15, 55-60.

Ornitz, E.M., \& Pynoos, R.S. (1989). Startle modulation in children with posttraumatic stress disorder. American Journal of Psychiatry, 146, 866-870.

Peeke, H.V.S., \& Petrinovich, L. (1984). Habituation, Sensitization, and Behavior. Orlando, Academic Press.

Pijlman, F.T., Herremans, A.H., van de Kieft, J., Kruse, C.G., \& van Ree, J.M. (2003). Behavioural changes after different stress paradigms, prepulse inhibition increased after physical, but not emotional stress. European Neuropsychopharmacology, 13, 369-380. 
Pilz, P., \& Schnitzler, H. (1996) Habituation and sensitization of the acoustic startle response in rats, Amplitude, threshold, and latency measures. Neurobiology of learning and Memory, 66, 67-79.

Raven, J.C. (1976). Standard Progressive Matrices. Oxford: Oxford Psychologists Press.

Roskies, E, Seraganian, P., Oseasohn, R., Hanley, J.A., Collu, R., Martin, N., and Smilga, C. (1986). The Montreal Type A Intervention Project: Major findings. Health Psychology, 5, 45-69.

Russell, J.A., Weiss, A., \& Mendelsohn, G.A. (1989). Affect Grid: A single item scale of pleasure and arousal. Journal of Personality and Social Psychology, 57, 493502.

Salamone, J.D., Cousins, M.S., \& Snyder, B.J. (1997). Behavioral functions of nucleus accumbens dopamine, empirical and conceptual problems with the anhedonia hypothesis. Neuroscience and Biobehavioral Reviews, 21, 341-359.

Schicatano, E. J., \& Blumenthal, T. D. (1998). The effects of caffeine and directed attention on acoustic startle habituation. Pharmacology, Biochemistry, and Behavior, 59, 145-150.

Schmajuk, N. A., \& Larrauri, J.A. (2005). Neural network model of prepulse inhibition. Behavioral Neuroscience, 119, 1546-1562.

Schmajuk, N.A., Larrauri, J.A., De la Casa, L.G., \& Levin, E.D. (2009). Attenuation of auditory startle and prepulse inhibition by unexpected changes in ambient illumination through dopaminergic mechanisms Behavioural Brain Research, 197, 251-261.

Scholes, K.E., \& Martin-Iverson, M.T. (2009). Alterations to pre-pulse inhibition (PPI) in chronic cannabis users are secondary to sustained attention deficits. Psychopharmacology, 207, 469-484.

Schwarzkopf, S.B., McCoy, L., Smith, D.A., \& Boutros, N.N. (1993). Test-retest reliability of prepulse inhibition of the acoustic startle. Biological Psychology, 34, 896900.

Sommer, K., van der Molen, M.W., \& De Pascalis, V. (2016). BIS/BAS sensitivity and emotional modulation in a prepulse-inhibition paradigm, A brain potential study. Physiology \& Behavior, 154, 100-113. 
Sutton, S.K., Davidson, R.J., Donzella, B., Irwin, W., \& Dottl, D. (1997). Manipulating affective state using extended picture presentations. Psychophysiology, 34, 217-226.

Swerdlow, N.R., Braff, D.L., \& Geyer, M.A. (1999). Cross-species studies of sensorimotor gating of the startle reflex. Annals of the New York Academy of Sciences, $\underline{877}, 202-16$.

Swerdlow, N.R., Caine, S.B., Braff, D.L., \& Geyer, M.A. (1992). The neural substrates of sensorimotor gating of the startle reflex, a review of recent findings and their implications. Journal of Psychopharmacology, 6, 176-190.

Swerdlow, N.R., \& Geyer, M.A. (1998). Using an animal model of deficient sensorimotor gating to study the pathophysiology and new treatments of schizophrenia. Schizophrenia Bulletin, 24, 285-301.

Swerdlow, N.R., \& Geyer, M.A. (1999) Neurophysiology and neuropharmacology of short lead interval startle modification. In M.E. Dawson, A.M. Schell, \& A.H. Boehmelt (Eds.), Startle Modification, Implications for Neuroscience. Cognitive Science and Clinical Science (pp. 114-133). New York: Cambridge University Press.

Swerdlow, N.R., Keith, V.A., Braff, D.L., \& Geyer, M.A. (1991). Effects of spiperone, raclopride, $\mathrm{SCH} 23390$ and clozapine on apomorphine inhibition of sensorimotor gating of the startle response in the rat. The Journal of Pharmacology and Experimental Therapeutics, 256, 530-536.

Swerdlow, N.R., Paulsen, J., Braff, D.L., Butters, N., Geyer, M.A., \& Swenson, M.R. (1995). Impaired prepulse inhibition of acoustic and tactile startle response in patients with Huntington's disease. Journal of neurology, neurosurgery, and psychiatry, 58, 192-200.

Swerdlow, N. R., Stephany, N. L., Talledo, J., Light, G., Braff, D. L., Baeyens, D., \& Auerbach, P. P. (2005). Prepulse inhibition of perceived stimulus intensity: Paradigm assessment. Biological Psychology, 69, 133-147.

Talalaenko, A. N., Abramets, I. J., Stakhovski, Y. V., Shekhovtsov, A. A., Chernikov, A. V., \& Shevchenko, S. L. (1994). The role of dopaminergic mechanisms on the brain in various models of anxious states. Neuroscience, Behavior, and Physiology, 24, 284-288. 
Thorne, G.L., Dawson, M.E., \& Schell, A.M. (2005). Attention and prepulse inhibition, the effects of task-relevant, irrelevant, and no-task conditions. International Journal of Psychophysiology, 56, 121-128.

Tidey, J.W., \& Miczek, K.A. (1996). Social defeat stress selectively alters mesocorticolimbic dopamine release, an in vivo microdialysis study. Brain research, 721, 140-149.

Toulouse, P., \& Pieron, H. (1998). Toulouse-Piéron (Prueba Perceptiva y de Atención). Madrid, TEA.

Turetsky, B.I., Calkins, M.E., Light, G.A., Olincy, A., Radant, A., \& Swerdlow, N.R. (2007). Neurophysiological endophenotypes of schizophrenia, The viability of selected candidate measures. Schizophrenia Bulletin, 33, 69-94.

Van den Berg, C.L., Lamberts, R.R., Wolterink, G., Wiegant, V.M., \& Van Ree, J.M. (1998). Emotional and footshock stimuli induce differential long-lasting behavioural effects in rats; involvement of opioids. Brain Research, 799, 6-15.

Vanman, E. J., Boehmelt, A. H., Dawson, M. E., \& Schell, A. M. (1996). The varying time courses of attentional and affective modulation of the startle eyeblink reflex. Psychophysiology, 33, 691-697.

Vrana, S.R., Spence, E.L., \& Lang, P.J. (1988). The startle probe response, a new measure of emotion? Journal of Abnormal Psychology, 97, 487-491.

Wrzesniewski, K. (1983). Emotional patterns in stress situations and somatic diseases. In C.D. Spielberger, R. Diaz-Guerrero, and J. Strelau (Eds.), Cross-cultural anxiety (Vol. 4), pp.21-31. Washington, DC: Hemisphere/Harper and Row

Wynn, J.K., Dawson, M.E., Schell, A.M., McGee, M., Salvesona, D., \& Green, M.F. (2004). Prepulse facilitation and prepulse inhibition in schizophrenia patients and their unaffected siblings. Biological Psychiatry, 55, 518-523.

Zhang, J., Forkstam, C., Engel, J.A., \& Svensson, L. (2000). Role of dopamine in prepulse inhibition of acoustic startle. Psychopharmacology, 149, 181-188. 
Table 1. Mean scores and Standard Deviations for pre- and post-experimental arousal and affect variables from the Mood Grid as a function of Groups for Experiments 1 (upper section) and 2 (lower section). 1 means maximum unpleasantness / minimum arousal, 5 neutral affect / medium arousal, and 9 maximum pleasantness / maximum arousal.

\begin{tabular}{ccccc}
\hline \multicolumn{5}{c}{ EXPERIMENT 1 } \\
\hline GROUP & Arousal Pre & Arousal Post & Affect Pre & Affect Post \\
\hline Stress & $6.18(1.40)$ & $7.82(.60)$ & $6.73(1.10)$ & $4.27(1.10)$ \\
\hline Control & $6.45(1.13)$ & $6.36(1.5)$ & $6.64(1.03)$ & $5.91(1.04)$ \\
\hline GROUP & Arousal Pre & Arousal Post & Affect Pre & Affect Post \\
\hline High load & $6.09(1.87)$ & $6.45(2.16)$ & $6.36(1.29)$ & $5.82(1.25)$ \\
\hline Low load & $6.09(1.51)$ & $5.27(2.24)$ & $6.27(1.10)$ & $5.64(1.63)$ \\
\hline
\end{tabular}




\section{Figure Captions.}

Figure 1. Mean pre-test startle magnitude (collapsed across trials) and mean startle magnitude for the 12 Pulse-alone experimental trials as a function of Group (Stress and Control). Error bars represent SEMs

Figure 2: Mean PPI percent collapsed across trials for each lead interval condition (40 ms vs. 60 ms vs. $80 \mathrm{~ms}$ ) as a function of Group (Stress and Control). Error bars represent SEMs

Figure 3: Mean pre-test mean startle magnitude (collapsed across trials) and mean startle magnitude for the 12 Pulse-alone experimental trials as a function of Group (Low Load task vs. High Load task). Error bars represent SEMs

Figure 4: Mean PPI percent collapsed across trials for each lead interval condition (40 ms vs. 60 ms vs. 80 ms) as a function of Group (Low Load task vs. High Load task). Error bars represent SEMs 


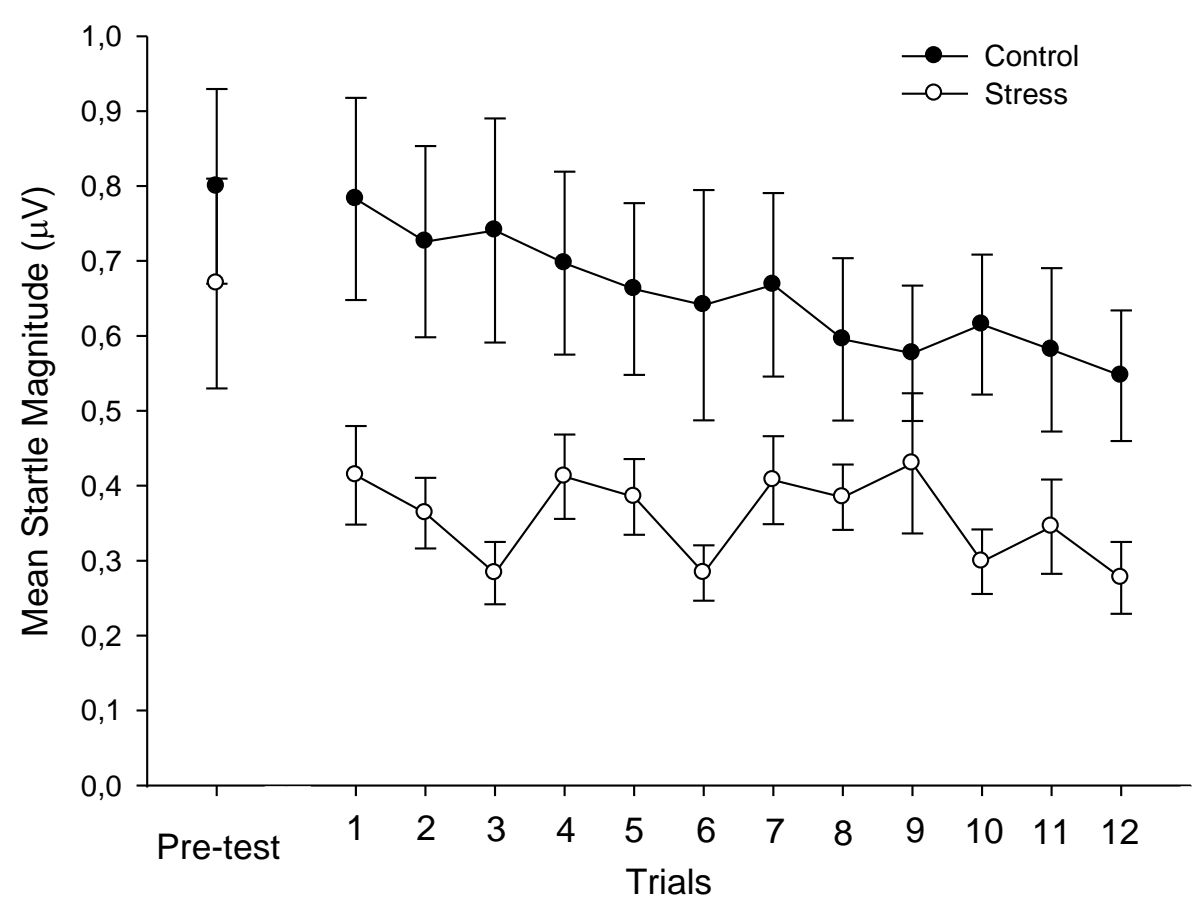

Figure 1. 


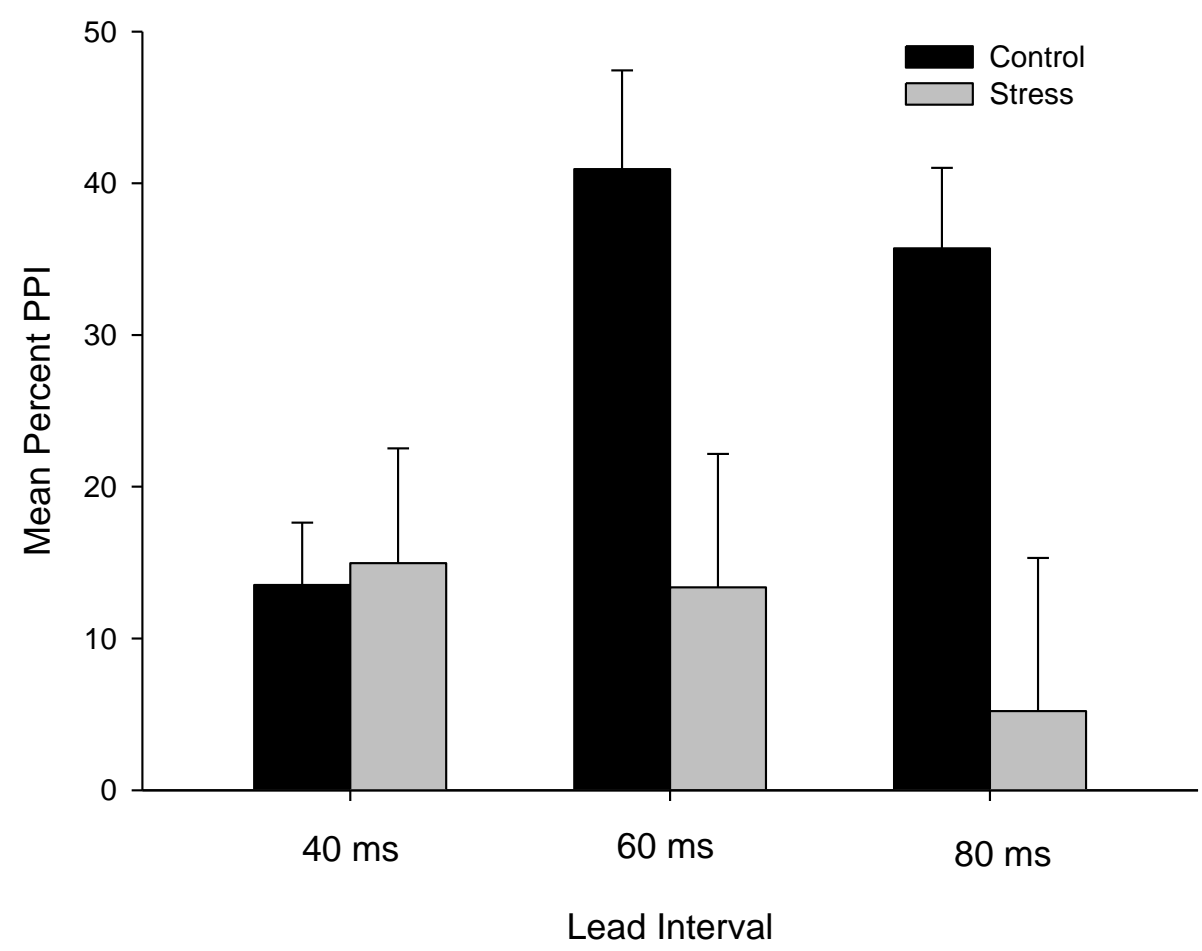

Figure 2. 


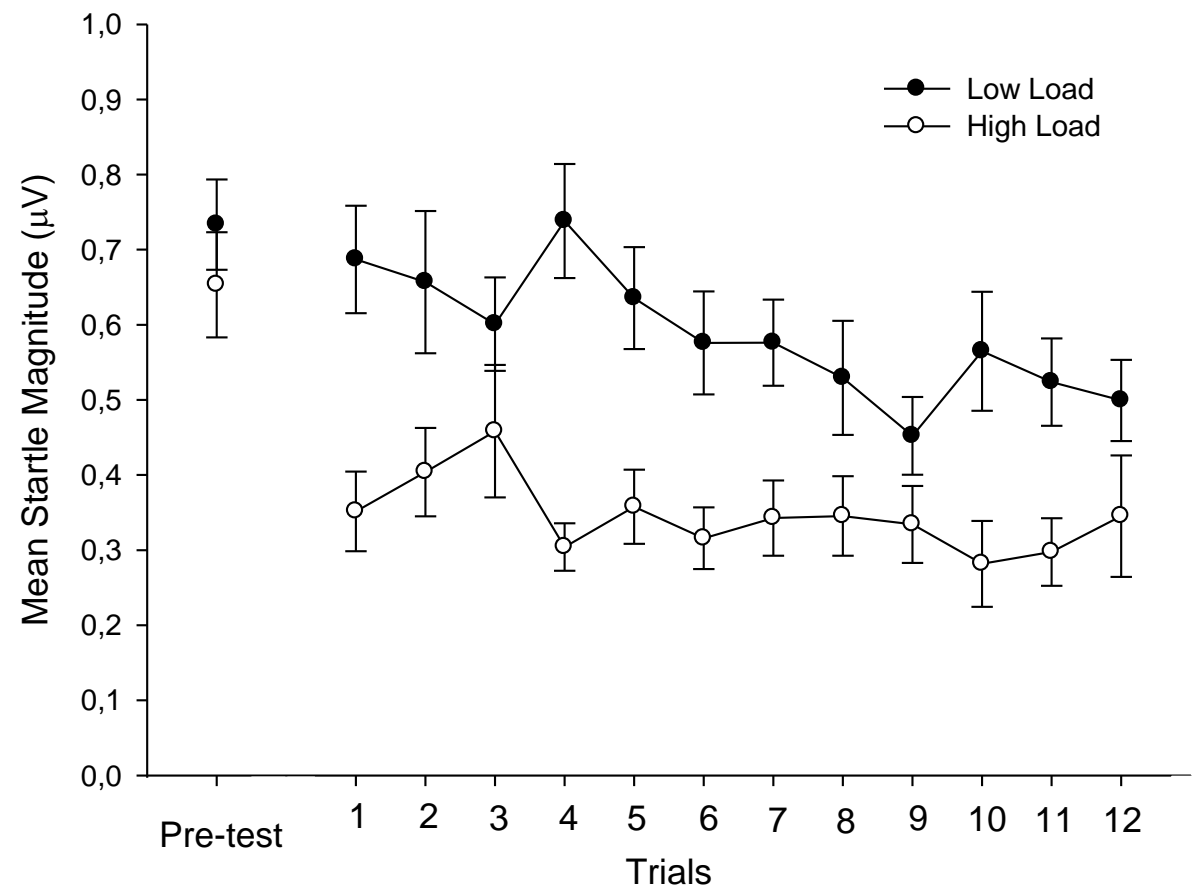

Figure 3. 


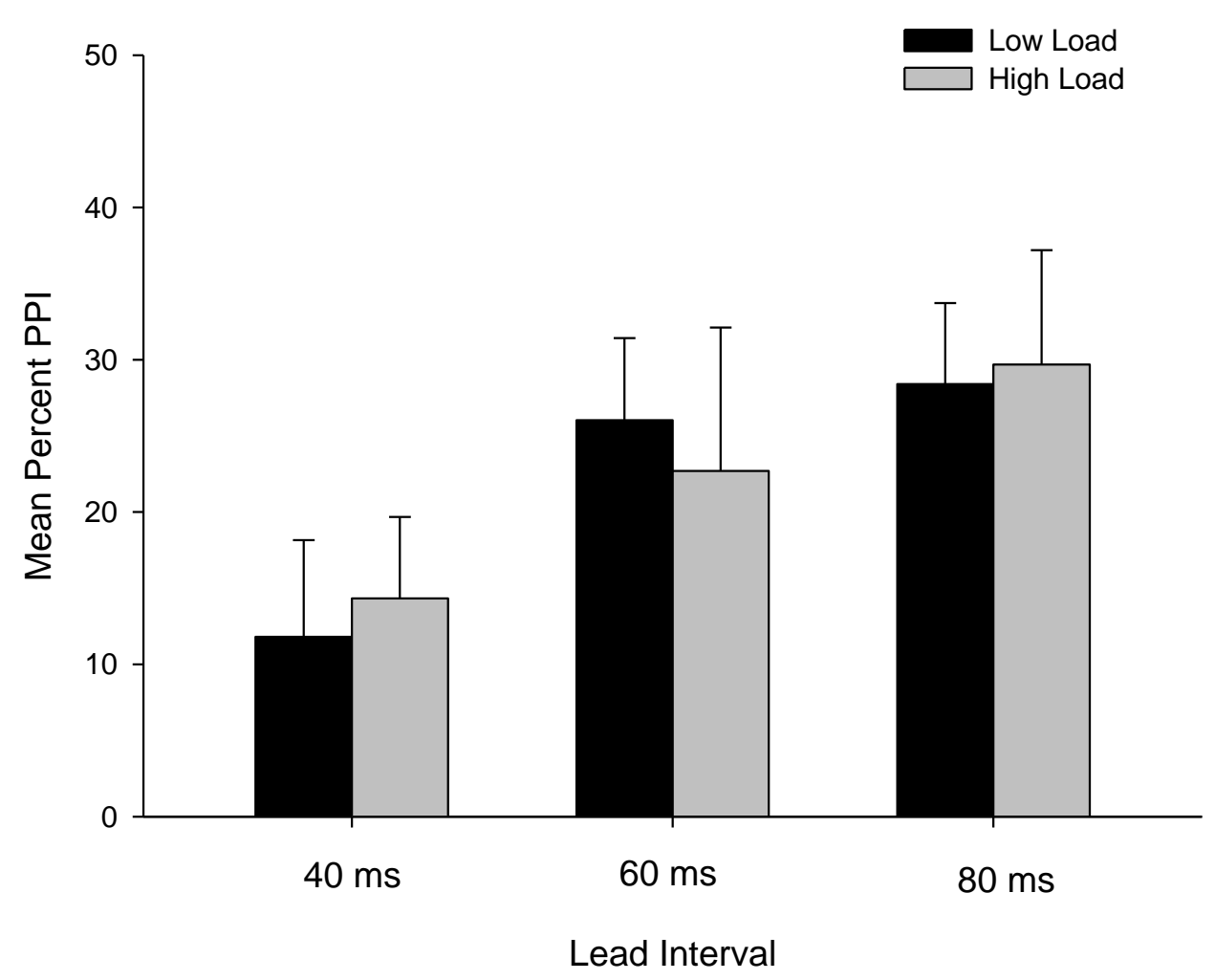

Figure 4. 